\title{
Coherent Control of Population Transfer in Rydberg Atoms via Chirped Microwave Pulses ${ }^{\dagger}$
}

\author{
Juan J. Carrera ${ }^{\ddagger, \S}$ and Shih-I Chu*,,\$ \\ Department of Chemistry, University of Kansas, Lawrence, Kansas 66045, and Center for Theoretical Sciences, \\ Department of Physics, National Taiwan University, Taipei 10617, Taiwan
}

Received: April 29, 2007; In Final Form: August 3, 2007

\begin{abstract}
We present a comprehensive and ab initio nonperturbative investigation of the coherent population transfer among the 3D high-lying Rydberg hydrogen and alkali atomic states via linearly polarized chirped microwave pulses. The time-dependent Schrödinger equation for the dynamical evolution of Rydberg atoms is accurately and efficiently solved by means of the time-dependent generalized pseudospectral method. For atomic $\mathrm{H}$, the population transfer from $n=35$ to 30 with nearly $100 \%$ efficiency is achieved by means of the sequential two-photon $\Delta n=-1$ transitions. The calculation fully utilizes all of the available orbital angular momentum $l$ states for a given $n$, and the interference pattern and population evolution dynamics of individual $l$ states are analyzed in detail. It is shown that the coherent control of the population transfer from the high $n$ to the low $n$ states can be accomplished by the optimization of the chirping parameters and microwave field strength. Similar analysis is performed for the $\mathrm{Na}$ atom, where the alkali atomic structure is described by an accurate model potential. We found that while the global population transfer pattern is qualitatively similar, there are significant differences in the dynamical response of atomic $\mathrm{H}$ and $\mathrm{Na}$ to the chirped microwave fields. Due to the degeneracy of the $l$ states (for a given $n$ ) in unperturbed atomic $\mathrm{H}$, the population transfer involves significant coupling and interference among a number of low-lying $l$ states. For the case of Na atoms, however, the population transfer from the $n$ to $(n-1)$ state is dominated by a single channel, namely, from the $\mid n, l=$ $0\rangle$ to the $|n-1, l=0\rangle$ state.
\end{abstract}

\section{Introduction}

Significant advances in laser technology in the last two decades have led to an increasing interest in the study of Rydberg wavepackets as a means to understanding and controlling quantum phenomena. ${ }^{1}$ Rydberg electron wavepackets are spatially localized quantum states of an electron possessing both particle-like and wave-like properties. The initial impetus in the study of electronic wavepackets arose from the desire to understand the limit of the classical-quantum correspondence principle in atomic systems. The study of quantum mechanics in regimes where the classical motion is chaotic is one of the most intriguing fundamental problems in atomic and molecular physics. $^{2,3}$ Recent rapid technological advancement in ultrafast laser technology has shifted the emphasis from the observation of atomic wavepackets toward their control. For example, wavepacket control of the principle quantum number $n^{4}$ or the parabolic quantum number $k^{5}$ or the control of both radial and angular evolution ${ }^{6}$ has been proposed for quantum information processing. Recently frequency- or amplitude-chirped pulses have also been used to perform quantum control of physical and chemical processes by robustly driving transitions between quantum states. 7,8

Adiabatic rapid passage (ARP) via chirped pulses is a robust and coherent method to transfer population from one quantum state to the other with essentially $100 \%$ efficiency. The requirement is that the frequency of the radiation coupling the two concerned states is swept through the resonance at a rate

\footnotetext{
† Part of the "Sheng Hsien Lin Festschrift".

* To whom correspondence should be addressed. E-mail: sichu@ ku.edu.

$\doteqdot$ University of Kansas.

$\S$ National Taiwan University.
}

smaller than the square of the Rabi frequency. Indeed, coherent population transfer via ARP in atoms through two-photon ${ }^{9}$ or multiphoton ${ }^{10}$ transitions using chirped pulses has been recently demonstrated. By exposing lithium Rydberg atoms to a chirped microwave pulse, robust transfer of population to the lower Rydberg states has been achieved successfully. ${ }^{9}$ Coherent population transfer consists of changing the principle quantum number $n$ through a passing sequence of $n$ states by a series of ARPs. These passages are characterized by the fact that there is no single sequence of levels but rather a sequence of a group of levels, namely, all of the orbital angular momentum $l$ states $(l=0,1,2 \ldots, n-1)$ for a fixed azimuthal angular momentum quantum number $m$, which are coupled together, assuming the microwave field is linearly polarized.

$\mathrm{Ab}$ initio 3D quantum studies of the Rydberg dynamics are intrinsically sophisticated and computationally challenging. The difficulties stem from the fact that Rydberg dynamics involve a very large spatial extent, long-time propagation of the timedependent Schrödinger equation in space and time, and the existence of the Coulomb singularity at the origin; all of these cannot be adequately treated by the conventional time-propagation methods ${ }^{11}$ using equal-spacing spatial grid discretization. Indeed, most of the theoretical studies of Rydberg atom dynamics in the past decade were limited to the one-dimensional or two-dimensional models. The recent development of the timedependent generalized pseudospectral (TDGPS) method, ${ }^{12}$ allowing nonuniform and optimal spatial discretization of the Hamiltonian and fast time propagation of the wave function, has been demonstrated to be capable of performing accurate $3 \mathrm{D}$ calculations of Rydberg atom spectroscopy ${ }^{13}$ as well as atomic $^{12,14-16}$ and molecular ${ }^{17-19}$ multiphoton processes in intense laser fields. In this paper, we extend the TDGPS method 
for the nonperturbative investigation of the coherent population transfer of 3D Rydberg atoms driven by chirped microwave pulses. The motivation of this paper is twofold. First, we study the effect of microwave-range chirped pulses in the coherent control of population transfer in Rydberg atoms. The study of the use of a chirped microwave pulse to change the principal quantum number $n$ of Rydberg atomic states is a subject of much current experimental interest. ${ }^{9,10,20}$ Second, we probe the interference dynamics and pattern arising from different $l$ states during the sequence of $\Delta n=-1$ population transfer from the high $n$ to the lower $n$ states. We perform the electron population transfer study for the Rydberg hydrogen and sodium atoms. As is to be shown, the responses of the $\mathrm{H}$ and alkali atoms to the chirped microwave pulses are qualitatively different, particularly for the lower $l$ states.

\section{Time-Dependent Hamiltonian and Numerical Method for Probing Rydberg Atom Dynamics}

The dynamics of the Rydberg $\mathrm{H}$ atoms is studied by solving accurately and efficiently the time-dependent Schrödinger equation (TDSE) in space and time (in atomic units)

$$
i \frac{\partial}{\partial t} \psi(\mathbf{r}, t)=\hat{H}(\mathbf{r}, t) \psi(\mathbf{r}, t)=\left[\hat{H}_{0}(\mathbf{r})+\hat{V}(\mathbf{r}, t)\right] \psi(\mathbf{r}, t)
$$

Here, $\hat{H}_{0}$ is the field-free Hamiltonian of atomic hydrogen, $\hat{H}_{0^{-}}$ $(\mathbf{r})=-1 / 2 \nabla^{2}-1 / \mathbf{r}$, and $\hat{V}(\mathbf{r}, t)$ is the time-dependent atomfield interaction. The Hamiltonian for the alkali atoms will be discussed in section IV. For a linearly polarized microwave field $(\mathbf{F} \mid \hat{z}), \hat{V}(\mathbf{r}, t)$ can be expressed in the length form as

$$
\hat{V}(\mathbf{r}, t)=-\mathbf{F} \cdot \mathbf{r} E(t)=-F z f(t) \sin [\Phi(t)]
$$

where $\mathbf{F}$ is the electric field amplitude, $f(t)$ is the pulse shape of the microwave field, and $\Phi(t)$ is the instantaneous phase of the microwave field. We adopt the form of the instantaneous phase of Conover et al. ${ }^{21}$

$$
\Phi(t)=\omega_{0} t+\beta \tau \ln \left[\cosh \frac{t-t_{0}}{\tau}\right]
$$

where $\omega_{0}$ is the carrier frequency of the microwave pulse. The chirp of the microwave field is determined by the instantaneous frequency

$$
\omega(t)=\frac{\mathrm{d} \Phi(t)}{\mathrm{d} t}=\omega_{0}+\beta \tanh \left(\frac{t-t_{0}}{\tau}\right)
$$

The chirp form can be controlled by adjusting the two parameters $\beta$ (in au) (for controlling the frequency sweeping range) and $\tau$ (in au) (for controlling the steepness of the chirped function). The parameter $t_{0}(\mathrm{au})$ is commonly set at the middle of the sweep. Typical parameters used in the numerical integration are $\beta=3.7 \times 10^{-6} \mathrm{au}, t_{0}=3.0 \times 10^{9} \mathrm{au}$, and $\tau=$ $5.0 \times 10^{9}$ au. Equation 1 is solved accurately and efficiently by means of the time-dependent generalized pseudospectral (TDGPS) method. ${ }^{12}$ The numerical scheme of the TDGPS method involves the following two essential steps: (a) The generalized pseudospectral (GPS) technique ${ }^{22}$ is used for nonuniform and optimal spatial discretization of the radial coordinate. This discretization is characterized by denser grids near the nuclear origin and sparser grids for larger distances. By mapping the semi-infinite domain $\left[0, r_{\max }\right]$ onto a finite domain $[-1,1]$ using a nonlinear transformation $r=r(x)=$ $L(1+x) /(1-x+\alpha)$, where $L$ and $\alpha=2 L / R_{\max }$ are both radial mapping parameters, the Coulomb singularity at $r=0$ and the corresponding long-range potential are satisfactorily resolved. (b) For the efficient time propagation of the wave function, a second-order split-operator technique in the energy representation, ${ }^{12}$ which allows the explicit elimination of undesirable fastoscillating high-energy components, is employed

$$
\begin{aligned}
& \psi(\mathbf{r}, t+\Delta t) \simeq \exp \left(-i \hat{H}_{0} \Delta t / 2\right) \times \exp (-i \hat{V}(r, \theta, t+ \\
&\Delta t / 2) \Delta t) \times \exp \left(-i \hat{H}_{0} \Delta t / 2\right) \psi(\mathbf{r}, t)+O\left(\Delta t^{3}\right)
\end{aligned}
$$

The wave function $\psi(\mathbf{r}, t)$ is propagated for a half-time step $\Delta t / 2$ in the energy representation. The atom-field interaction is performed in the coordinate representation for a time step $\Delta t$. Last, the wave function is transformed back to the energy representation and propagated for another $\Delta t / 2$. The initial Rydberg state $\psi(\mathbf{r}, t=0)=\psi_{n=35, l=0, m=0}(\mathbf{r})$ is then propagated for the pulse duration of 20000 optical cycles (o.c.). The microwave field is slowly ramped as in the form of eq 6

$$
f(t)= \begin{cases}\sin ^{2} \frac{\pi t}{20 T} & t \leq 3000 T \\ 1 & t \geq 3000 T\end{cases}
$$

where $T=2 \pi / \omega_{0}$

Once the time-dependent wave function $\psi(\mathbf{r}, t)$ is determined, the population evolution for a given $n l$ state under the influence of a microwave chirped field will be given by

$$
p_{n l}(t)=\left|\left\langle\psi_{n l}(\mathbf{r}, 0) \mid \psi(\mathbf{r}, t)\right\rangle\right|^{2}
$$

The total population from all of the $l$ states for a given $n$ state at a given time $t$ is computed by summing all of the $l$ contributions from the time-dependent population $p_{n l}(t)$

$$
p_{n}(t)=\sum_{l}^{l_{\max }} p_{n l}(t)=\sum_{l}^{l_{\max }}\left|\left\langle\psi_{n l}(\mathbf{r}, 0) \mid \psi(\mathbf{r}, t)\right\rangle\right|^{2}
$$

where $l_{\max }$ is the maximum number of partial waves used. To achieve a fully numerical convergence, we use up to 300 radial grid points to describe the spatial range of $5000 \mathrm{au}$, and $l_{\max }=$ 36 partial waves.

\section{Population Transfer of Atomic Hydrogen as a Function of the Chirp and Microwave Field Strength}

The transition probability of the electron to move from the $|n, l\rangle$ to $\left|n-1, l^{\prime}\right\rangle$ state is related to the slew rate of the chirped frequency. To achieve complete population transfer from state to state, the ratio of the instantaneous frequency to the time span must be small enough to allow atoms to completely pass over the avoided crossings at the corresponding two-photon resonance frequency. We define the slew rate of our pulses by $\Delta \omega / \Delta t(\mathrm{GHz} / \mathrm{ns})$. The microwave instantaneous frequency is chirped over a frequency interval from 75.6 to $84.2 \mathrm{GHz}$ or $\Delta \omega / 2 \pi=8.6 \mathrm{GHz}$. Such frequency sweeping corresponds to the two-photon resonance transition between $n=35$ and 34 . Once the frequency range is fixed, the time interval $\Delta t$ is varied between 4.3 and $17.2 \mathrm{~ns}$. The corresponding slew rate is on the order from 0.5 to $2.0 \mathrm{GHz} / \mathrm{ns}$. We compute the transition probabilities for three microwave field strengths, $F=0.5,1.0$, and $2.0 \times 10^{-8}$ au as shown in Figure $1 \mathrm{a}-\mathrm{c}$, respectively. In the case of the weaker fields, Figure 1a, the population transfer from $n=35$ to 34 does not occur completely for any slew rate. As the field strength of the microwave field is increased to 1.0 $\times 10^{-8}$ au, nearly complete transfer to $n=34$ begins to take place if the slew rate is greater than $0.5 \mathrm{GHz} / \mathrm{ns}$, as shown in Figure 1b. By further increasing the field strength, as in the 

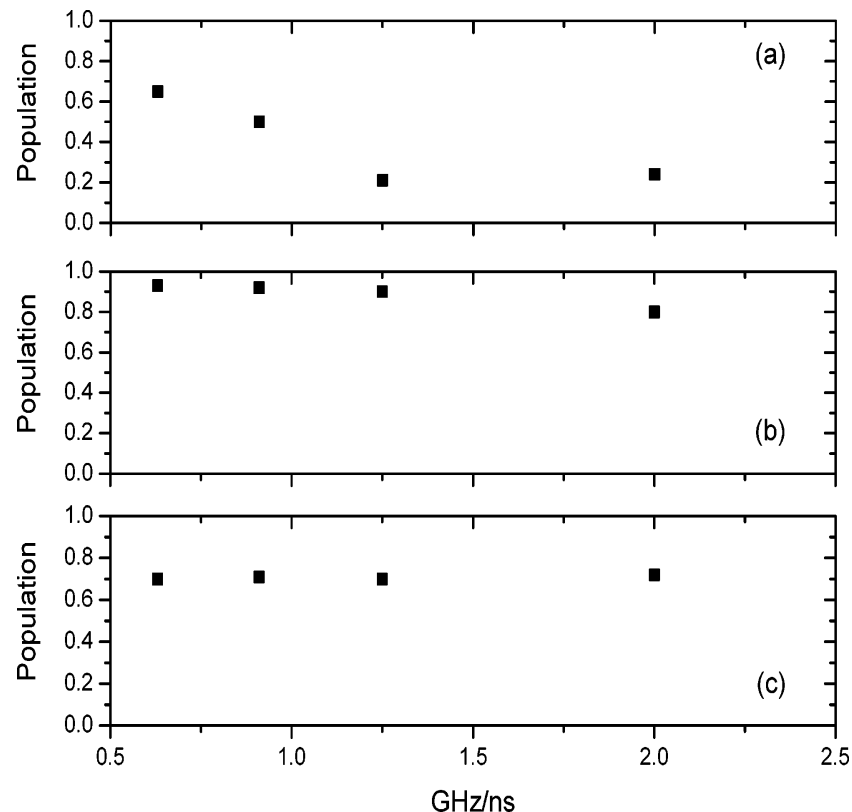

Figure 1. Total transition probabilities of Rydberg $\mathrm{H}$ atoms from the $n=35$ to 34 states as a function of the slew rate $\Delta \omega / \Delta t(\mathrm{GHz} / \mathrm{ns})$ for three microwave field strengths, (a) $5 \times 10^{-9}$, (b) $1.0 \times 10^{-8}$, and (c) $2.0 \times 10^{-8}$ au.

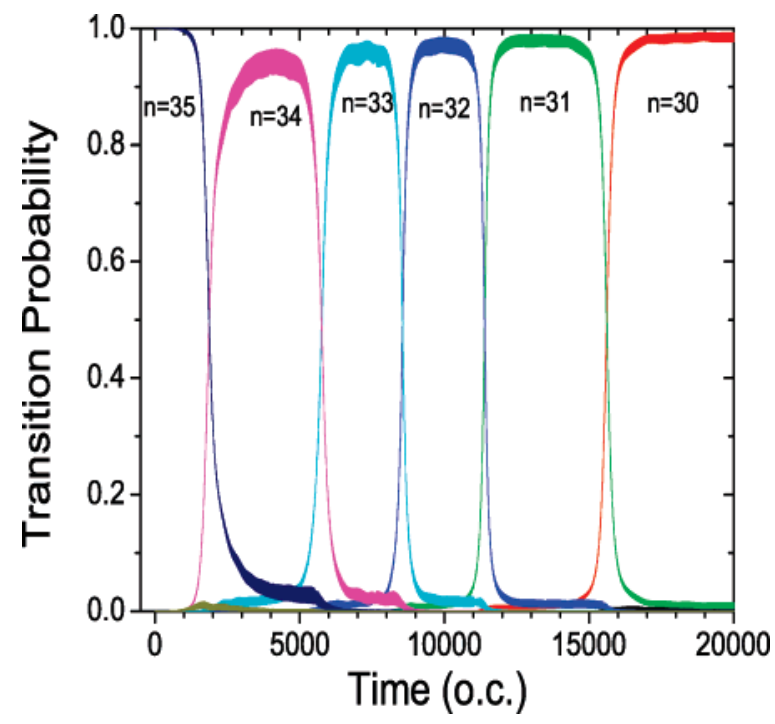

Figure 2. Transition probability evolution of Rydberg hydrogen atoms initially located in the principal quantum state $n=35$ and driven sequentially to the $n=30$ state via a chirped microwave pulse. The microwave field strength used is $F=1.1 \times 10^{-8}$ au, and the chirp parameters used are $\beta=3.7 \times 10^{-6} \mathrm{au}, t_{0}=3.0 \times 10^{9} \mathrm{au}$, and $\tau=$ $5.0 \times 10^{9}$ au.

case of Figure $1 \mathrm{c}$, to $2.0 \times 10^{-8}$ au, full population transfer does not take place even in the case of large slew rates. Hence, to coherently control the population transfer, the field strength as well as the rate of change of the instantaneous frequency must be optimized.

Now, we study the effects of frequency swept pulses on the population transfer from the $n=35$ to 30 state for the hydrogen atom case. Population transfer via chirped microwave pulses is attained at the two-photon resonance between adjacent states, namely, $\Delta n=-1$. Moreover, due to the electric dipole selection rules, the two-photon coupling will only link states with $\Delta l=$ \pm 2 . In our case, the initial state is prepared at the $\mid n=35, l=$ $m=0\rangle$ state. Hence, the dynamical evolution will be confined to the manifold of even- $l$ states. The magnetic quantum number

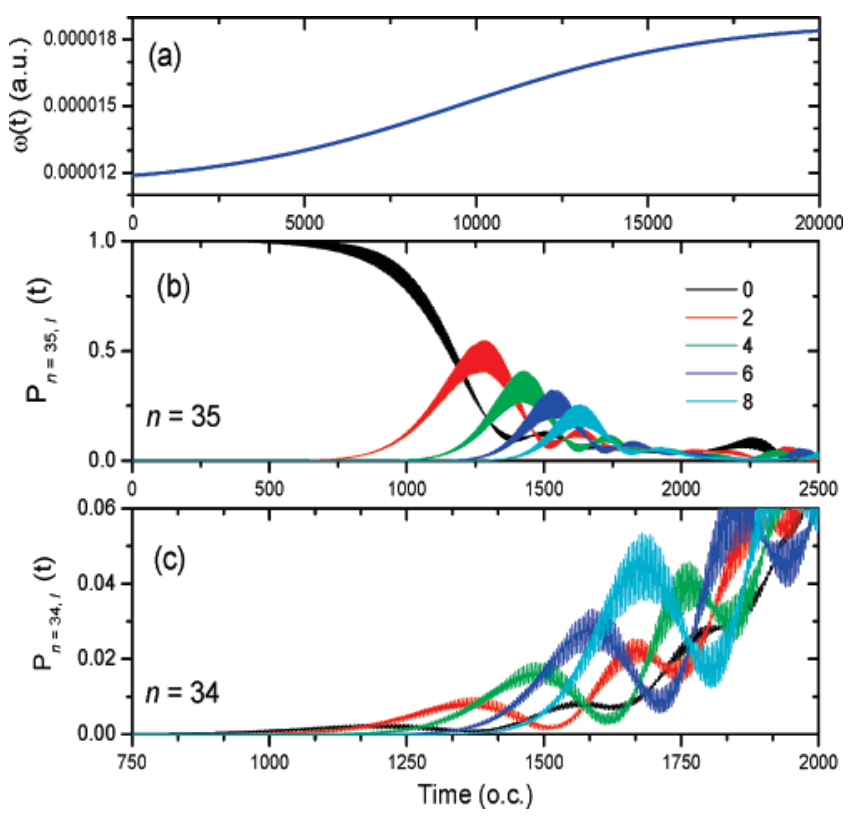

Figure 3. (a) The chirped microwave pulse frequency dependence. (b) Depopulation of the initial state $|n=35, l=0\rangle$ to higher- $l$ states for Rydberg hydrogen atoms. (c) Initial population transitions to the $|n=34, l\rangle$ state. The microwave field strength used is $F=1.1 \times 10^{-8}$ $\mathrm{au}$, and $\beta=3.7 \times 10^{-6} \mathrm{au}$.
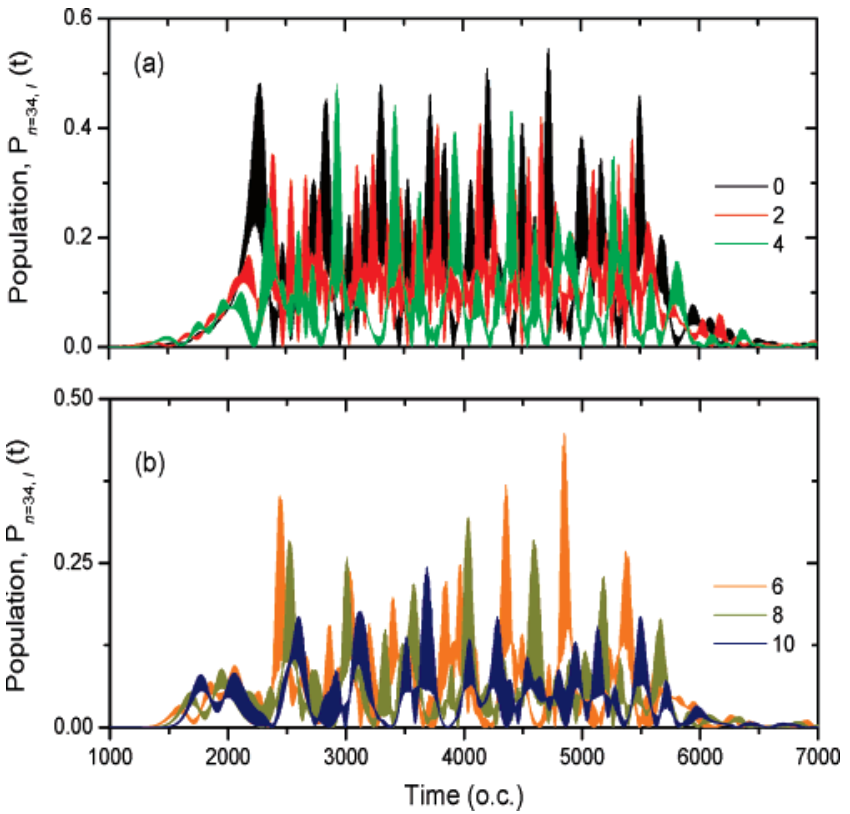

Figure 4. The dynamic population evolution of an individual angular momentum $l$ component of the $n=34$ manifold for (a) $l=0,2,4$ and (b) $l=6,8,10$ in a chirped microwave field. The microwave field parameters are the same as those in Figure 3.

$m$ is a good number, and thus, $\Delta m=0$. Figure 2 shows the nearly complete sequential population transitions from $n=35$ to 30. The instantaneous frequency of the pulse, as shown in Figure 3a, is positively chirped and tuned to follow the spacing between the $\Delta n=-1$ resonances. The initial frequency is located near the $n=35$ to 34 two-photon resonance transition, and the frequency chirp stops right after reaching the $n=31$ to 30 resonance. The frequency sweeping range is from $1.18 \times$ $10^{-5}$ to $1.81 \times 10^{-5}$ au (or from 77.6 to $119.1 \mathrm{GHz}$ ). The population transfer stops at the state $n=30$.

Figure $3 \mathrm{~b}$ illustrates in more detail the gradual depletion of the initial $n=35, l=0$ state as well as the time evolution of 


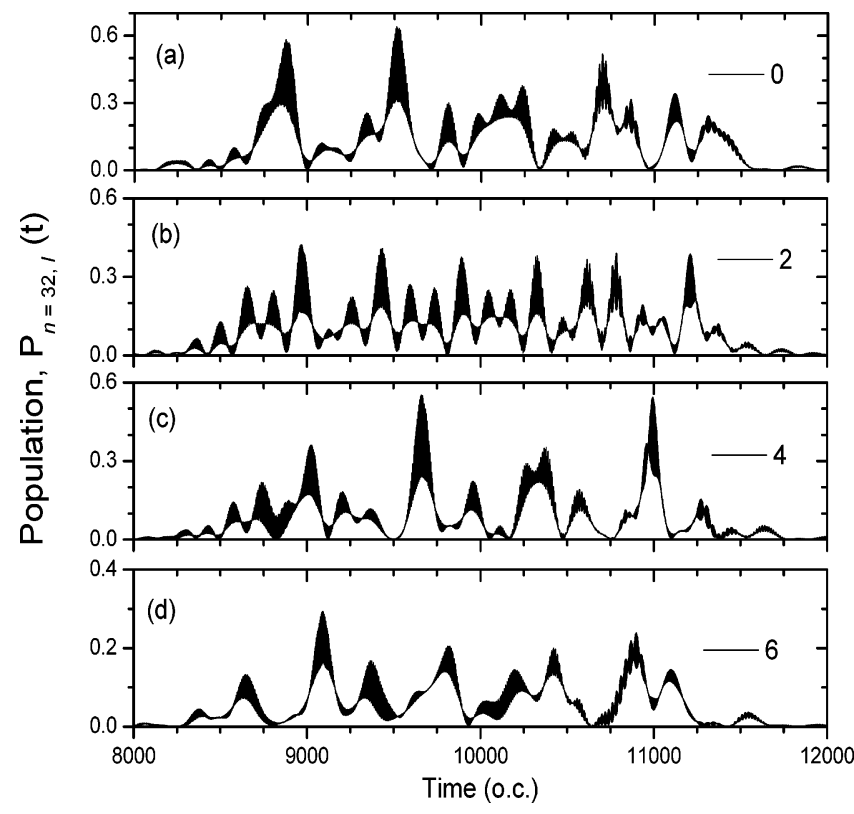

Figure 5. The dynamical evolution of the most dominant angular momentum $l$ components of the $n=32$ state for (a) - (d) for $l=0,2$, 4 , and 6 , respectively. The microwave field parameters are the same as those in Figure 3.

TABLE 1: Model Potential Parameters for Na (in au)

\begin{tabular}{lccccccc}
\hline$l$ & $\alpha$ & $r_{\mathrm{c}}$ & $S$ & $A_{1}$ & \multicolumn{1}{c}{$A_{2}$} & \multicolumn{1}{c}{$B_{1}$} & $B_{2}$ \\
\hline 0 & 0.946 & 2.09 & 3.4776465 & -4.4987218 & -0.77850759 & 2.50 & 1.25 \\
1 & 0.946 & 2.09 & 2.7448107 & -6.8685263 & -0.46055194 & 2.1 & 1.05 \\
$>1$ & 0.946 & 2.09 & 0.21570440 & -3.5100571 & -0.015635093 & 1.90 & 0.95
\end{tabular}

the population from low- $l$ to higher- $l$ states within the $n=35$ manifold. We note that the transition probability to the lowlying $l$ states is considerably larger than those of the high- $l$ states. Moreover, all of the coupled $l$ states are characterized as having one significant peak which occurs consecutively as $l$ increases. As the first $\Delta n=-1$ adiabatic passage to the $n=34$ manifold is taking place, the population of the individual $l$ state in the $n$ $=34$ manifold exhibits oscillatory population patterns, as shown Figure 3c. Furthermore, the peak population of the higher-lying $l$ states is larger than those of lower- $l$ states. Consequently interferences among all of the coupled $l$ channels begin to emerge and become more important.

Figure $4 \mathrm{a}$ and $\mathrm{b}$ shows the population evolution of the principal channels $l=0,2,4$ and $l=6,8,10$, respectively, of the $n=34$ manifold after the completion of the population transfer from the $n=35$ to 34 state. The population migrates periodically from low- $l$ to high- $l$ angular momentum states and vice versa. Low- $l$ states are, on average, more populated. Indeed, only the lowest six coupled $l$ states are the most dominant channels during the population transition from $n=35$ to 30 . However, the $l$ states' population revivals due to the interference among all of the possible coupling paths are not necessarily in sequence. Figure $5 \mathrm{a}-\mathrm{d}$ displays a characteristic case of the population time evolution pattern corresponding to the individual $|n=32, l\rangle$ state, where $l=0,2,4,6$. Note that all of these low-lying $l$ states are significantly coupled and populated, and the number of population revivals is different for each $l$ state. We note that for Rydberg $\mathrm{H}$ atoms in linearly polarized microwaves, $\Delta m=0$, only two quantum numbers $n$ and $l$ are required to describe the population transfer process. Rigorously speaking, one channel calculation in spherical coordinates is not realistic since it does not lead to any physical transitions. Two channel calculations $(l=0,1)$ are the simplest possible way to induce population transfer, and it is also the closest one to the 1D calculation. For atomic $\mathrm{H}$, our results in Figures $3-5$ show clearly that multichannel $(l=0,2,4,6,8,10)$ calculations are necessary to get the results fully converged. That means that a $1 \mathrm{D}$ calculation for atomic $\mathrm{H}$ will not give the quantitatively correct results. Similar observations were reported in one of our previous papers ${ }^{23}$ in which the dynamical evolution of multiphoton ionization of Rydberg $\mathrm{H}$ atoms was studied using the generalized Floquet approach. We found that there is large discrepancy between the $1 \mathrm{D}$ and $3 \mathrm{D}$ results for a certain range of microwave field strengths and frequencies. In that regime, the $3 \mathrm{D}$ calculation leads to more efficient ionization than that of the 1D calculation. We expect similar behavior to take place in the present analysis of population transfer.

\section{Population Transfer in Rydberg Na Atoms Driven by Chirped Microwave Pulses}

In this section, we study the population transfer in Rydberg alkali atoms. An accurate one-electron model potential for the description of the sodium (Na) atoms will be adopted. ${ }^{24}$ Model potential methods have been widely used to calculate atomic ${ }^{25}$ and molecular properties ${ }^{26}$ for one- and two-valence electron systems as well as photoionization processes. ${ }^{27} \mathrm{~A}$ salient feature of the model potential consists of describing the valenceelectron-core interaction with the correct long-range polarization terms. In addition, the parameters that determine the shortrange part of the potential are chosen so that experimental spectra and low-energy electron scattering phase shifts are reproduced to very high accuracy for all angular momentum symmetries.

TABLE 2: Comparison of the Calculated Na Energies with the Experimental Values (in au). For Each Angular Momentum $l$, Two Rows of Energies $E(n, l)$ are Listed; the Upper Row Refers to Calculated Model-Potential Results, and the Lower Row Refers to the Experimental Values

\begin{tabular}{cccccccc}
\hline \multicolumn{7}{c}{$E(n, l)$} \\
\cline { 2 - 7 }$l / n$ & 3 & 4 & 5 & 6 & 7 & 8 & -0.011304 \\
\hline 0 & -0.188860 & -0.071572 & -0.037582 & -0.023131 & -0.015661 & -0.008541 \\
& -0.188862 & -0.071579 & -0.037585 & -0.023132 & -0.015662 & -0.011304 & -0.008541 \\
1 & -0.111541 & -0.050931 & -0.029194 & -0.018919 & -0.013254 & -0.009800 & -0.007540 \\
& -0.111550 & -0.050935 & -0.029195 & -0.018920 & -0.013254 & -0.009800 & -0.007539 \\
2 & -0.055942 & -0.031444 & -0.020106 & -0.013952 & -0.010245 & -0.007840 & -0.006192 \\
& -0.055938 & -0.031443 & -0.020106 & -0.013953 & -0.010245 & -0.007841 & -0.006193 \\
3 & & -0.031268 & -0.020011 & -0.013896 & -0.010209 & -0.007816 & -0.006175 \\
& & -0.031269 & -0.020011 & -0.013896 & -0.010209 & -0.007816 & -0.006175 \\
4 & & & -0.020002 & -0.013890 & -0.010205 & -0.007813 & -0.006173 \\
& & & -0.020002 & -0.013890 & -0.010205 & -0.007813 & -0.006173
\end{tabular}




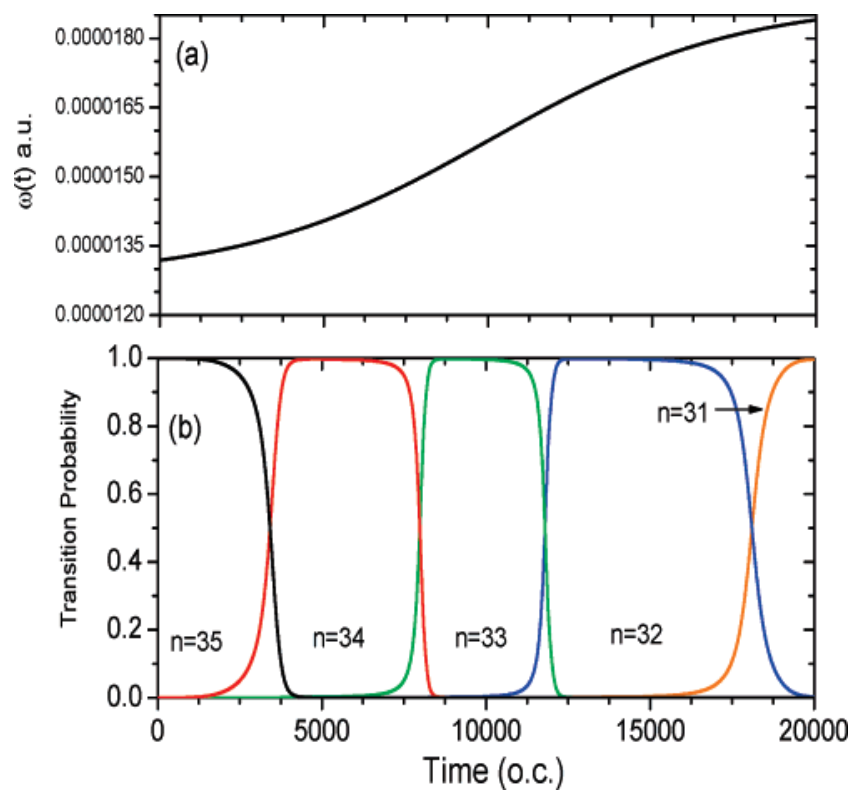

Figure 6. (a) The chirped microwave pulse frequency dependence. (b) Transition probability of $\mathrm{Na}$ Rydberg atoms initially located in the $|n=35, l=0\rangle$ state followed by sequential robust transitions to the $n$ $=31$ state. The microwave field strength is $1.1 \times 10^{-10}$ au, $\beta=3.0 \times$ $10^{-6} \mathrm{au}, t_{0}=4.0 \times 10^{9} \mathrm{au}$, and $\tau=3.0 \times 10^{9} \mathrm{au}$.

TABLE 3: Sodium Energy Values Corresponding to the Population-Transferred States (in au). Note that $E(n-1) p$ is Very Close to the Halfway Energy Between $E(n s)$ and $E(n-$ 1) $s$

\begin{tabular}{ccc}
\hline$n$ & $l=0$ & $l=1$ \\
\hline 35 & -0.00044141 & -0.00042885 \\
34 & -0.00046886 & -0.00045512 \\
33 & -0.00049895 & -0.00048387 \\
32 & -0.00053203 & -0.00051545 \\
31 & -0.00056851 & -0.00055021
\end{tabular}

The angular-momentum-dependent model potential has the following form

$$
\begin{aligned}
V_{l}(r)=-\frac{\alpha}{2 r^{4}} W_{6}\left(\frac{r}{r_{\mathrm{c}}}\right)-\frac{1}{r}-\left(\frac{N-S}{r}+A_{1}\right) \mathrm{e}^{-B_{1} r}- \\
\\
\left(\frac{S}{r}+A_{2}\right) \mathrm{e}^{-B_{2} r}
\end{aligned}
$$

where $N=10$ is the number of core electrons, $\alpha$ is the $\mathrm{Na}^{+}$ core dipole polarizability, and $W_{n}$ is a core cutoff function given by

$$
W_{n}(x)=1-\left[1+n x+\frac{(n x)^{2}}{2 !}+\ldots+\frac{(n x)^{n}}{n !}\right] \mathrm{e}^{-n x}
$$

where $r_{\mathrm{c}}$ is the effective core radius for $\mathrm{Na}^{+}$. There are two potentials for $\mathrm{Na}$, one for the s state $l=0$ and one for higher angular momenta states $(l=1,2,3, \ldots)$. The values of the parameters are listed in Table 1 . Table 2 presents the boundstate energies for sodium atoms predicted by the model potentials and their corresponding experimental values. The agreement is excellent, demonstrating the accuracy of the model potential used.

The TDSE, eq 1 , is reformulated for the $\mathrm{Na}$ atoms in the presence of linearly polarized (LP) microwave fields. Here, $\hat{H}_{0}$ is the field-free Hamiltonian for the $\mathrm{Na}$ atoms

$$
\hat{H}_{0}(\mathbf{r})=-\frac{1}{2} \nabla^{2}+\sum_{l}\left|Y_{l}^{0}\right\rangle V_{l}(\mathbf{r})\left\langle Y_{l}^{0}\right|
$$
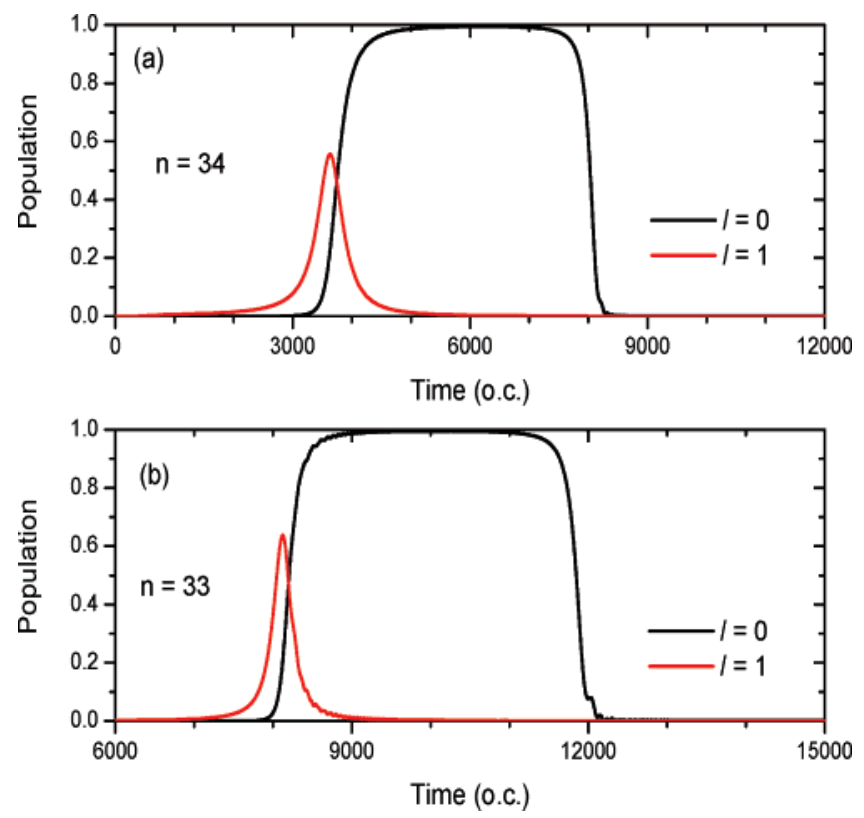

Figure 7. (a) Transition probabilities of Na Rydberg atoms initially located in the $|n=35, l=0\rangle$ state moving to the $|n=34, l=1\rangle$ state and then to the $|n=34, l=0\rangle$ state. (b) As time goes by, the population then transfers to $|n=33, l=1\rangle$ and then to the $|n=33, l=0\rangle$ state. Note that in both cases, only the $n s$ and $n p$ states have significant populations. The microwave field strength used is $1.1 \times 10^{-10}$ au, $\beta=$ $3.0 \times 10^{-6} \mathrm{au}, t_{0}=4.0 \times 10^{9} \mathrm{au}$, and $\tau=3.0 \times 10^{9} \mathrm{au}$.

where the model potential $V_{l}(r)$ is described in eq 9 and $Y_{l}^{0}$ is a spherical harmonic function. The atom-field coupling and the form of the chirp frequency resembles to that of eqs 2 and 4, respectively. The time propagation of the wave function is performed by means of the TDGPS method outlined in a previous section from which the transition probabilities to a given $n l$ state are determined similarly as for the hydrogen atom case.

To obtain full population transfer from higher $n$ state to lower $n$ states, the frequency chirp range for the case of the $\mathrm{Na}$ atoms is taken from $1.32 \times 10^{-5}$ to $1.83 \times 10^{-5}$ au (or from 86.8 to 120.4 GHz), as seen in Figure 6a. Also, the optimal microwave field strength to reach the full population transitions for $\mathrm{Na}$ atoms is found to be $1.0 \times 10^{-10}$ au. Figure $6 \mathrm{~b}$ shows that complete population transfer from the $n=35$ state down to $n$ $=31$ can be achieved. Since the $n p$ states are located nearly halfway between the $n s$ and $(n-1) s$ states, the two-photon $\Delta n$ $=-1$ transitions are in close resonance with the one-photon transitions, namely, the $n s$ to $(n-1) p$ and $(n-1) p$ to $(n-1) s$ transitions. Table 3 shows the energies corresponding to the $n$ $=35$ to 31 states for $l=0$ and $l=1$, respectively. Figure 7 a and $\mathrm{b}$ shows the population transfer dynamics for $n=34$ and 33 , respectively. Note that only $l=0$ and 1 states have significant populations, and the lowest angular momentum state $l=0$ is largely predominant during the time evolution process. This state has little mixing with other higher $l>1$ states. In fact, our calculation shows that $98 \%$ of the transitions occur between the $|n, l=0\rangle$ and $|n-1, l=0\rangle$ transitions. On the other hand, in the case of Rydberg hydrogen atoms, several low- $l$ states are strongly coupled and significantly populated during the dynamical evolution of the Rydberg system. This is the main difference between the alkali and hydrogen atoms. The degeneracy of the $|n, l\rangle$ states for a given $n$ leads to significant coupling and interference among different $l$ states in atomic hydrogen. 


\section{Conclusion}

We have presented an ab initio nonperturbative study of the interaction of microwave frequency swept (chirp) pulses with Rydberg atoms using TDGPS. An optimal slew rate and microwave field strength are the main attributes necessary to coherently drive the population transfer. We found that complete population transfer can be obtained by chirping the frequency of the microwave field from $n=35$ to 30 for the case of the hydrogen atom and from $n=35$ to 31 for the case of Na atom. This demonstrates that it is possible to achieve coherent control of the population transfer of a Rydberg system by optimizing the chirped pulses. Periodic revival in population is observed in any of the $n$ manifold states. For the hydrogen atom case, the population revivals are caused by the interferences among all of the $l$ states of a given $n$ state. Nevertheless, only the lowest six coupled $l$ states $(l=0,2,4,6,8,10)$ are found to be the dominant channels during the population transitions. In the case of $\mathrm{Na}$ atoms, due to the large quantum defect for low- $l$ states, only the $|n, l=0\rangle$ to $|n-1, l=0\rangle$ channel dominates the population transitions.

Acknowledgment. This work is partially supported by the Chemical Sciences, Geosciences and Biosciences Division of the Office of Basic Energy Sciences, Office of Sciences, U.S. Department of Energy, and by the U.S.-NSF and by National Taiwan University.

\section{References and Notes}

(1) Fielding, H. H. Annu. Rev. Phys. Chem. 2005, 56, 91.

(2) Gutzwiller, M. C. Chaos in Classical and Quantum Mechanics; Springer-Verlag: New York, 1990.
(3) Koch, P. M.; van Leeuwen, K. A. H. Phys. Rep. 1995, 255, 289. 463.

(4) Ahn, J.; Weinacht, T. C.; Bucksbaum, P. H. Science 2000, 287,

(5) Ahn, J.; Hutchinson, D. N; Rangan, C.; Bucksbaum, P. H. Phys. Rev. Lett. 2001, 86, 1179.

(6) Rangan, C.; Ahn, J.; Hutchinson, D.; Bucksbaum, P. H. J. Mod Opt. 2003, 49, 2239.

(7) Batista, A. A.; Citrin, D. S. Phys. Rev. B 2006, 74, 195318.

(8) Gibson, G. N. Phys. Rev. A 2005, 72, 041404. 053413

9) Lambert, J.; Noel, M. W.; Gallagher, T. F. Phys. Rev. A 2002, 66

(10) Maeda, H.; Gurian, H. J.; Norum, D. V. L.; Gallagher, T. F. Phys. Rev. Lett. 2006, 96, 073002.

(11) Kulander, K., Ed. Special Issue on Time-Dependent Methods for Quantum Dynamics. Comput. Phys. Commun. 1991, 63, 1.

(12) Tong, X. M.; Chu, S. I. Chem. Phys. 1997, 217, 119.

(13) Tong, X. M.; Chu, S. I. Phys. Rev. A 2000, 61, 031401.

(14) Tong, X. M.; Chu, S. I. Phys. Rev. A 2000, 61, 021802.

(15) Tong, X. M.; Chu, S. I. Phys. Rev. A 2001, 64, 013417.

(16) (a) Carrera, J. J.; Tong, X. M.; Chu, S. I. Phys. Rev. A 2006, 74 023404. (b) Carrera, J. J.; Tong, X. M.; Chu, S. I. Phys. Rev. A 2005, 71, 063813.

(17) Zhou, Z. Y.; Chu, S. I. Phys. Rev. A 2005, 71, 011402.

(18) (a) Chu, X.; Chu, S. I. Phys. Rev. A 2001, 63, 023411. (b) Chu, X.; Chu, S. I. Phys. Rev. A 2001, 64, 063404.

(19) Chu, X.; Chu, S. I. Phys. Rev. A 2004, 70, 061402.

(20) Maeda, H.; Norum, D. V. L.; Gallagher, T. F. Science 2005, 307, 1757.

(21) Conover, C. W. S.; Doogue, M. C.; Struwe, F. J. Phys. Rev. A 2002, 65, 033414

(22) Yao, G.; Chu, S. I. Chem. Phys. Lett. 1993, 204, 381.

(23) Wang, K.; Chu, S. I. Phys. Rev. A 1989, 39, 1800.

(24) Chu, X.; Chu, S. I. Phys. Rev. A 2001, 64, 013406.

(25) Hansen, J. E.; Laughlin, C.; van der Hart, H. W.; Verbockhaven, G. J. Phys. B 1999, 32, 2099.

(26) Bottcher, C.; Dalgarno, A. Chem. Phys. Lett. 1975, 36, 137.

(27) Butler, K.; Mendoza, C. J. Phys. B 1996, 29, 1767. 